\title{
Engaging Undergraduates in Crystallographic Research: "Structure of the Yersinia pestis UDP-glucose-1-phosphate uridylyltransferase"
}

\author{
George T. Lountos ${ }^{1,2}$, Morgan E. Gibbs ${ }^{2}$ and David S. Waugh ${ }^{2}$ \\ ${ }^{1}$ Basic Science Program, Frederick National Laboratory for Cancer \\ Research sponsored by the National Cancer Institute, ${ }^{2}$ Macromolecular \\ Crystallography Laboratory, National Cancer Institute, Frederick, MD \\ 21702
}

The NIH Summer Internship Program in Biomedical Research (SIP) provides undergraduates the opportunity to spend a summer working in an NIH lab under the supervision of a scientist. The program lasts approximately 8-12 weeks and is sponsored by the NIH Office of Intramural Training \& Education. Here, we describe our experience with the SIP program and present a training module designed to introduce undergraduate students to various aspects of crystallographic research including molecular cloning, protein construct design and expression, purification by various chromatographic techniuues, crystallizaation of macromolecules, X-ray difraction and structure solution and refinement. As a case study, a review of the literature was performed to select a protein of biomedical interest and the student solved the structure within the summer training period. In this case, the Yersinia pestis UDP-glucose-1-phosphate uridylyltransferase (UDP-glucose pyrophosphorylase), a potential anti- plague drug target was investigated. The research project demonstrated the interdisciplinary breadth of crystallographic research and its important role in structure-based drug design research. 